\title{
Multi-domain active sound control and noise shielding
}

\author{
H. $\operatorname{Lim}^{\mathrm{a})}$ \\ Acoustics Research Centre, University of Salford, Salford, Greater Manchester, M5 4WT, United Kingdom \\ S. V. Utyuzhnikov \\ School of Mechanical, Aerospace and Civil Engineering, University of Manchester, M13 9PL, United Kingdom \\ Y. W. Lam \\ Acoustics Research Centre, University of Salford, Salford, Greater Manchester, M5 4WT, United Kingdom
}

A. Turan
School of Mechanical, Aerospace and Civil Engineering, University of Manchester, M13 9PL, United Kingdom

(Received 16 April 2010; revised 15 November 2010; accepted 2 December 2010)

\begin{abstract}
This paper describes an active sound control methodology based on difference potentials. The main feature of this methodology is its ability to automatically preserve "wanted" sound within a domain while canceling "unwanted" noise from outside the domain. This method of preservation of the wanted sounds by active shielding control is demonstrated with various broadband and realistic sound sources such as human voice and music in multiple domains in a one-dimensional enclosure. Unlike many other conventional active control methods, the proposed approach does not require the explicit characterization of the wanted sound to be preserved. The controls are designed based on the measurements of the total field on the boundaries of the shielded domain only, which is allowed to be multiply connected. The method is tested in a variety of experimental cases. The typical attenuation of the unwanted noise is found to be about $20 \mathrm{~dB}$ over a large area of the shielded domain and the original wanted sound field is preserved with errors of around $1 \mathrm{~dB}$ and below through a broad frequency range up to $1 \mathrm{kHz}$. (C) 2011 Acoustical Society of America. [DOI: 10.1121/1.3531933]
\end{abstract}

PACS number(s): 43.50.Ki, 43.40.Sk, 43.55.Dt, 43.55.Br [KVH] Pages: 717-725

\section{INTRODUCTION}

Active control of sound is a technique for altering acoustic fields to a desired one by introducing controllable active secondary sound sources called controls. An example problem formulation in this area involves a given region of space (bounded or unbounded) to be shielded from unwanted external noise by the active controls. The controls establish an active boundary, shielding the region from the noise. This specific strategy for noise cancellation by means of active boundary controls is called active shielding (AS). The overall problem of active noise cancellation becomes more complicated if, along with the unwanted noise, a wanted sound component is present inside the protected region.

Generally, existing conventional active control methods, for example those developed by Nelson and Elliott, ${ }^{1}$ and Kincaid et al., ${ }^{2,3}$ require an accurate description of the original noise source in order to devise a global cancellation solution. When the measurement is carried out in close proximity of the shielded domain and the noise source is not measured, significant noise reduction can generally be achieved only locally in conventional approaches. ${ }^{1}$ Often in practice it is not feasible to measure the physical values of the original noise source since the noise source is not always accessible. ${ }^{4}$ In addition, the transfer function of the sound through the problem domain has to be taken into account to achieve a global noise cancellation solution. This is particu-

\footnotetext{
a) Author to whom correspondence should be addressed. Electronic mail: h.lim@edu.salford.ac.uk
}

larly difficult if the medium of propagation is inhomogeneous. To overcome these limitations and associated practical difficulties, the difference potential method (DPM) proposed here can provide a convenient solution. The theoretical concept is based on the method described in Refs. 5 and 6. It allows one to obtain a general solution to the AS problem for arbitrary geometries, properties of the medium, or boundary conditions.

Theoretically, the DPM allows us to reduce a boundary value problem set in a complex domain to a boundary equation. Its key characteristics include the capability to cancel out the unwanted noise in a large region of the shielded domain, while requiring no detailed knowledge of either the sound transfer function for the problem domain or the noise sources. The only input data needed by the methodology are acoustic quantities at the perimeter of the protected region (in practice they can be measured). By requiring only this limited input data, the unique characteristics of the method can provide a practical and cost-effective control system. Moreover, these quantities may pertain to an overall acoustic field composed both of unwanted and wanted components. The methodology automatically differentiates between the two. The method suggested by Jessel and Mangiante, ${ }^{7,8}$ and Canevet, ${ }^{9}$ hereby called the JMC method, ${ }^{10}$ yields solutions for global noise cancellation in a similar way when only the unwanted noise is present in the protected domain. The main difference between the approaches based on the DPM and JMC is that only the former provides the advantages of preservation of the wanted sound and volumetric noise cancellation through an entire shielded domain when the total field 
composed of both the wanted sound and noise is measured on the boundary. In addition, the DPM-based approach allows a shielded domain to be multiply connected. This capability is potentially very useful for applications related to noise control and room acoustics, as it enables protection of the predefined space against the noise coming from the outside, while at the same time not interfering with the ability of the listener to listen to wanted sound or communicate inside the room. Although this technique has been introduced and studied theoretically in previous publications, ${ }^{11,12}$ the unique feature that allows us to preserve the wanted sounds in multi-domain has never been experimentally studied and published in literature. The main focus of the paper is an implementation of the novel AS technique based on the difference potentials to multi-domain tests with broadband signals. In practice a sound field is generally composed of broadband frequencies rather than a pure tone. A broadband sound source may cause extreme fluctuations of sound pressure at some frequencies due to resonances and anti-resonances in a duct closed by rigid terminations. Quite often a control system based on many other conventional AS methods fails to achieve efficient cancellation of noise at resonances. The reason is that the sensitivity of their solutions to errors is too high at resonances. With that in mind, the characteristics and practical limitations of the approach over a broad frequency band will be evaluated and discussed in this paper. As we are in an early stage of the experimental investigation for the method, a real-time control system has not been implemented yet. The overall system is assumed to be linear time-invariant and repeatable in the experiment.

\section{THEORETICAL FORMULATION}

Details of the theoretical formulation have already been described in previous publications. ${ }^{13,14}$ Only a brief outline of the concept is given here to help the understanding relevant to the experimental design. Assume that the propagation of sound is governed by a linear partial differential equation or system in a domain $D_{o}$. The sound field is composed of both adverse noise and wanted sound. We formulate the AS problem as follows. It is required to find such additional sources that the solution to the modified problem coincides with the wanted sound in a subdomain $D$, i.e. $D$ is part of $D_{o}$ that is to be shielded. It is important to note here that the domain $D$ is not necessarily simply connected. It is also noted that the reverberation field of wanted sound is also considered as part of the wanted sound. From a practical point of view, the additional sources, which are the "controls," cannot be immediately realized because the solution assumes the distribution of controls to be continuous. Here, a discrete distribution of the controls can be obtained via the theory of difference potentials (see Refs. 15 and 16 for details). It can also be interpreted as a discrete approximation of the continuous solution. For the AS solution it is sufficient to have access only to the trace of the total acoustic field on the boundary of the domain $D$. In particular, no knowledge of the actual sources (wanted and unwanted) is required. Thus, such active controls are more practical then the trivial solution of having a control equals to the ideal negative of the unwanted source, which is difficult to implement even if the adverse sources are explicitly available.

It is important to emphasize that the control sources are obtained for the general case and do not require knowledge of the Green's function of the problem. It has been shown that the space AS solution is based on the knowledge of the total sound pressure and the normal component of the particle velocity on boundary surface of the shielded domain. ${ }^{15,16}$ The general solution is applicable in the general case of 3D flow field.

The solution can be illustrated for a one-dimensional case, in which the primary noise sources are situated in the area $-\ell<x<\ell$, whereas the secondary (control) sources $G$ are to be placed at $x=-\ell$ and $x=\ell$ to protect the domains $x<-\ell$ and $x>\ell$. These domains are interpreted as subdomains of a single multiply connected domain $D$. We suppose the field is monochromatic and, in the frequency domain, the generated wanted field is represented by sound pressure $\mathrm{A}_{1} e^{-j k x}$ if $x<-\ell$ and $\mathrm{A}_{2} e^{j k x}$ if $x>\ell$. In turn, it is assumed that the noise is generated in such a way that field $\mathrm{B}_{1} e^{j k x}$ propagates toward domain $x<-\ell$, while field $\mathrm{B}_{2} e^{-j k x}$, toward domain $x>\ell$. For simplicity, anechoic terminations are assumed in the example so that there are no reflections from the ends. It should be noted that the general solution method itself can be applied to cases with any arbitrary terminations.

Let us put both a monopole and dipole at each of the two points $x=-\ell$ and $x=\ell$. Assume that the amplitudes of the monopoles and dipoles are given by $q_{i}$ and $b_{i}$, respectively, where $i=1$ corresponds to $x=-\ell$, while $i=2$ in the case of $x=\ell$. In addition, suppose both the dipoles are oriented toward $x=0$.

The field generated by all the primary and secondary sources is given by

$$
\begin{aligned}
\bar{p}(x)= & \mathrm{A}_{1} e^{-j k x}+\mathrm{A}_{2} e^{j k x}+\mathrm{B}_{1} e^{j k x}+\left(q_{1}-b_{1}\right) e^{j k(x+\ell)} \\
& +\left(q_{2}+b_{2}\right) e^{j k(x-\ell)}
\end{aligned}
$$

if $x<-\ell$ and

$$
\begin{aligned}
\bar{p}(x)= & \mathrm{A}_{1} e^{-j k x}+\mathrm{A}_{2} e^{j k x}+\mathrm{B}_{2} e^{-j k x}+\left(q_{1}+b_{1}\right) e^{-j k(x+\ell)} \\
& +\left(q_{2}-b_{2}\right) e^{-j k(x-\ell)}
\end{aligned}
$$

if $x>\ell$.

We require only the field $\bar{p}(x)=\mathrm{A}_{1} e^{-j k x}+\mathrm{A}_{2} e^{j k x}$ to be composed of the two wanted sounds in the protected domains $x<-\ell$ and $x>\ell$. It should be noted that this requirement is fundamentally different from that of an active absorber, which would have required only $\mathrm{A}_{1} e^{-j k x}$ in the domain $x<-\ell$ and $\mathrm{A}_{2} e^{j k x}$ in the domain $x>\ell$.

Then, we arrive at the following requirements,

$$
\left(q_{1}-b_{1}\right) e^{j k \ell}+\left(q_{2}+b_{2}\right) e^{-j k \ell}+\mathrm{B}_{1}=0
$$

if $x<-\ell$ and

$$
\left(q_{1}+b_{1}\right) e^{-j k \ell}+\left(q_{2}-b_{2}\right) e^{j k \ell}+\mathrm{B}_{2}=0
$$

if $x>\ell$. 
The particle velocity and sound pressure before the control are given by

$$
\begin{aligned}
& \bar{p}(-\ell)=\mathrm{A}_{1} e^{j k \ell}+\mathrm{A}_{2} e^{-j k \ell}+\mathrm{B}_{1} e^{-j k \ell}, \\
& \bar{u}(-\ell)=\frac{1}{\rho c}\left(\mathrm{~A}_{1} e^{j k \ell}-\mathrm{A}_{2} e^{-j k \ell}-\mathrm{B}_{1} e^{-j k \ell}\right), \\
& \bar{p}(\ell)=\mathrm{A}_{1} e^{-j k \ell}+\mathrm{A}_{2} e^{j k \ell}+\mathrm{B}_{2} e^{-j k \ell}, \\
& \bar{u}(\ell)=\frac{1}{\rho c}\left(\mathrm{~A}_{1} e^{-j k \ell}-\mathrm{A}_{2} e^{j k \ell}+\mathrm{B}_{2} e^{-j k \ell}\right) .
\end{aligned}
$$

Next, having formally applied the secondary sources derived in Ref. 15 for a simply connected domain, at each of the two boundary points $(x= \pm \ell)$, we arrive at the following controls,

$$
\begin{array}{ll}
q_{1}=\frac{\rho c \bar{u}(-\ell)}{2}, & q_{2}=-\frac{\rho c \bar{u}(\ell)}{2}, \\
b_{1}=\frac{\bar{p}(-\ell)}{2}, & b_{2}=\frac{\bar{p}(\ell)}{2} .
\end{array}
$$

Alternatively, the controls (4) can simply be postulated. Then, substituting Eqs. (3) into (4), one can verify that conditions (1) and (2) are valid.

To analyze the operation of the controls, let us consider, for example, the domain $x<-\ell$. From Eq. (1) one can obtain that the control sources at point $x=-\ell$ generate the following field,

$$
\bar{p}(x)=-\left\{\left(q_{2}+b_{2}\right) e^{j k(x-\ell)}+\mathrm{B}_{1} e^{j k x}+\mathrm{A}_{2} e^{j k x}\right\}+\mathrm{A}_{2} e^{j k x} .
$$

The above equation is written to show explicitly that the controls at $x=-\ell$ attenuate any field coming into domain $x<-\ell$, on the one hand, and restore the wanted field (the last term with $\mathrm{A}_{2}$ ) from the right-hand side, on the other hand.

Overall, the governing acoustics equations after the control are written as

$$
\begin{aligned}
& \frac{\partial p}{\partial t}+\rho c^{2} \frac{\partial u}{\partial x}=\rho c^{2} q_{\mathrm{vol}}+f_{p}, \\
& \frac{\partial u}{\partial t}+\frac{1}{\rho} \frac{\partial p}{\partial x}=\frac{b_{\mathrm{vol}}}{\rho}+f_{u},
\end{aligned}
$$

where the controls are given by

$$
\begin{aligned}
& q_{\mathrm{vol}}(x)=u(-\ell) \delta(x+\ell)-u(\ell) \delta(x-\ell), \\
& b_{\mathrm{vol}}(x)=p(-\ell) \delta(x+\ell)-p(\ell) \delta(x-\ell) .
\end{aligned}
$$

Here, $f_{p}$ and $f_{u}$ are the appropriate source functions, $\delta(x)$ is the 1D delta-function, determined in the space of distributions (see, e.g., Ref. 17). The controls $q_{\mathrm{vol}}$ and $b_{\mathrm{vol}}$ of Eq. (5) depend on the particle velocity and sound pressure, respectively. It is to be noted that, as soon as we introduce the delta-function, we should consider the solution to the problem in the generalized sense. ${ }^{18}$ Alternatively, the delta-function should be approximated by its counterpart in the space of standard functions.

One can see, the controls (5) are a partial case of the general solution ${ }^{19}$ in $3 \mathrm{D}$ case,

$$
\begin{aligned}
& q_{\mathrm{vol}}=u_{n}(\Gamma) \delta(\Gamma), \\
& \vec{b}_{\mathrm{vol}}=\vec{n} p(\Gamma) \delta(\Gamma) .
\end{aligned}
$$

Here, $\vec{n}$ is the external normal to the boundary $\Gamma$ of the protected domain and $\delta(\Gamma)$ is the delta-function assigned to the surface $\Gamma$. In the example, the coordinate of the normal to the boundary $\Gamma$ at $x=-\ell$ equals 1 , while at $x=\ell$ it is equal to -1 .

In practical applications, the point sources (5), should be approximated by spatially extended terms. ${ }^{15}$ For example, the controls at point $x=\ell$ is represented by

$$
\begin{aligned}
& q_{\mathrm{vol}, \ell}^{(h)}=-\frac{\Theta_{h}(x-\ell)}{h} u_{\ell}, \\
& b_{\mathrm{vol}, \ell}^{(h)}=-\frac{\Theta_{h}(x-\ell)}{h} p_{\ell} .
\end{aligned}
$$

Here, $\Theta_{h}(x) \equiv \theta(h / 2-x) \theta(h / 2+x)$, where $h$ denotes the finite difference step and $\theta(x)$ is the indicator function; the particle velocity $u_{\ell}$ and sound pressure $p_{\ell}$, respectively, should be measured near the point $x=\ell$. Accordingly, these controls are represented by the volume velocity per unit volume and the force per unit volume, see Ref. 1. In an experimental setting, $h$ in Eq. (7) corresponds to the thickness of the source, ${ }^{15,20}$ the control $q_{\mathrm{vol}}^{(h)}$ is implemented as an acoustic monopole and the control $b_{\mathrm{vol}}^{(h)}$ is implemented as a dipole. The thickness of the source should be adequately smaller than the wavelength. This follows from the theoretical approximation $k h \ll 1$. $^{15}$

In Ref. 15, for a simply connected domain it is shown that the controls (6) preserve the reverberation field of the wanted sound. The mechanism behind this is as follows. The controls attenuate any field coming into the domain to be shielded and, at the same time, generate only the field inside the domain that is exactly required to restore the reverberation of the wanted sound there. Thus, the AS controls are "transparent" to the reflected component of the wanted sound coming into the protected domain. The same conclusion is applicable to a multiply connected domain. For instance, in the example in question, assume that there is a rigid termination on the right-hand side at $x=\ell_{1}>\ell$. Then, we can interpret any reverberation of wanted sound generated in the domain $\ell<x<\ell_{1}$ as just the wanted sound from this domain. In addition, there is no reverberation of noise because of its attenuation in the domain $\ell \leq x \leq \ell_{1}$. Then, the problem can be reduced to the example considered above except the reverberation of the wanted sound generated in the domain $x<-\ell$. In contrast to the previous case, the input (measured) data at $x=-\ell$ change due to the contribution of the reflected field of the wanted sound from the left-hand side. However, as noted above, the appropriate additional secondary sources do not damage the reverberation field in domain $x<-\ell$. In turn, the controls at $x=\ell$ are transparent to the wanted field coming from the left-hand side and its reverberation holds inside the protected domain $\ell \leq x \leq \ell_{1}$.

In the experimental implementation of the AS solution, there are also some restrictions depending on the frequency of acoustic signals generated. In practice, to maximize the efficiency in attenuation in 3D space, the optimum distribution 
of the control sources on boundary surfaces has to be defined. Optimization of the control sources with respect to different criteria has been studied by Loncaric and Tsynkov in Ref. 21. It is to be noted that the described approach has recently been extended to a nonstationary formulation and arbitrary degree of sound control in Refs. 19 and 22, respectively.

\section{EXPERIMENTAL RESULTS}

\section{A. AS of a simply connected domain}

Regarding the validation of the AS solution based on difference potentials, it is helpful to start by analyzing a onedimensional linear problem in the first instance. The solution for the AS problems either with or without the wanted sounds have previously been experimentally validated with pure tone sound sources in a duct, and the results were reported in Ref. 20. Following this work, the experiment is now extended to cover broadband sound fields, including multiple resonance regions. This is done in a cylindrical duct manufactured of polypropylene tubing, which is sufficiently rigid to allow losses through the duct walls to be neglected. ${ }^{23}$ As most AS techniques are more effective in general at lower frequency rather than at higher frequency, ${ }^{1,24}$ the frequency range is limited to below $1 \mathrm{kHz}$ in the experiment. The duct is $4.42 \mathrm{~m}$ in length. Its inner diameter is $0.17 \mathrm{~m}$, which allows it to be approximated acoustically as onedimensional up to a frequency of about $1 \mathrm{kHz}$. Figure 1(a) illustrates the excitation of an unwanted noise source at the right-end of the duct, and wanted sound source inside of the shielded domain in a one-dimensional, rigid walled cylindrical enclosure. The domain to be shielded occupies approximately one third of the entire volume of the enclosure on the left-hand side. Any sound field having its acoustic source situated in the shielded domain is defined as "wanted." Sources outside are otherwise "unwanted" or "noise." The sound field includes reflections of the sound in the tube. In other words, the wanted field is the combination of the sound directly emitted from the wanted sound source and its reverberation within the tube. The fields of the wanted sound and noise, and the outputs of the controls satisfy the plane wave conditions. Measuring sensors and a control source unit are located at the boundary of the shielded domain, at $x=0$. The sound pressure is measured along the axis of the duct in all the experiments.

In the measuring process, before obtaining the AS solutions for a given problem, directional and non-directional components of the sound field are measured using a Brüel \& Kjær PULSE Sound \& Vibration analyzer with the control sources off. The former is the normal component of the par-

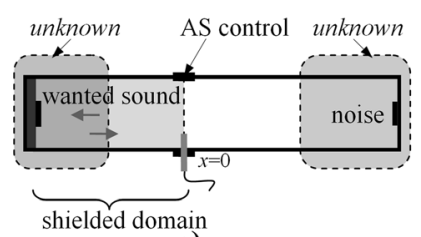

a)

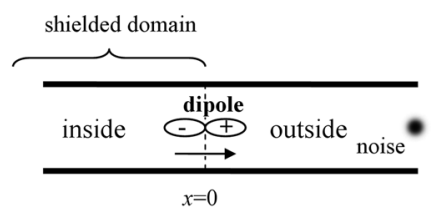

b)
FIG. 1. Experimental setup for the test: (a) noise cancellation and preservation of wanted sound and (b) direction of an acoustic dipole. ticle velocity $u_{o}$, and the latter is the acoustic pressure $p_{o}$ of the total field at the boundary. Then, the directional component measured defines a non-directional AS control source which is a monopole. On the other hand, the non-directional component measured is used to define a dipole control source which is directional.

The control sound field is derived from the measurements of the total field of the unwanted noise and the wanted sound on the boundary of the shielded domain. Unlike other approaches, for the preservation of a desirable sound and cancellation of noise, the procedure does not require any additional explicit information regarding the wanted sound or the system. In contrast, previous studies, e.g. Refs. 25 and 26 , for similar control cases required either the wanted sound or the unwanted noise to be absent in the measurement. In a recent paper, directional measuring devices, i.e. directional microphones, have been used in order to identify the wanted component apart from the total field. ${ }^{27}$ This is not required in our case because the wanted and unwanted components are discriminated automatically even in the case when the reverberation of the wanted sound propagates from the same direction of the unwanted one ${ }^{15}$ (see also example given in the Theoretical Formulation section). The measurement of the particle velocity at the boundary and the difference potential formulation are able to capture the difference in the location of the wanted and unwanted sources automatically. When the control sources are mounted on the boundary, the direction of the dipole source defines the inside and outside of a shielded domain. For this reason the direction of the dipole source must be perpendicular to the boundary and pointed out from the shielded domain [Fig. 1(b)]. The sound generation system consists of loudspeakers, amplifiers, and a PC with a multi-channel sound card. The measured values, adjusted for the transfer function of the signal generator, are used to calculate offline the control source signals based on the difference potential theory. The source strengths of the controls $b$ and $q$ related to the reference signal $V_{\text {ref }}$ are

$$
\hat{b}=\frac{\hat{p}_{o} A_{s}}{H_{d}}, \quad \hat{q}=\frac{\left(\hat{u}_{o} \vec{n}\right) A_{s}}{H_{m}} .
$$

Here $A_{s}$ is a cross-sectional surface area, $H_{d}$ is the transfer function of the dipole source signal generator, $H_{m}$ is the transfer function of the monopole source signal generator, and $\vec{n}$ is a unit normal vector on the boundary surface. In (8) $\hat{b}=b / V_{\text {ref }}, \hat{q}=q / V_{\text {ref }}, \hat{p}_{o}=p_{o} / V_{\text {ref }}$, and $\hat{u}_{o}=u_{o} / V_{\text {ref }}$.

Then, the control source signals (6) are then saved as phase-synchronous .wav files which can be played back using a multi-channel compatible wave editor. This calculation is not carried out in real-time in the current setup. The resulting attenuation of the unwanted sound and the preservation of the wanted sound are studied by measuring the total field composed with the wanted, unwanted, and control sound all together in the duct.

\section{B. Sensitivity analysis}

In the first instance, a quick demonstration of the ability of the AS method to attenuate broadband unwanted noise is 

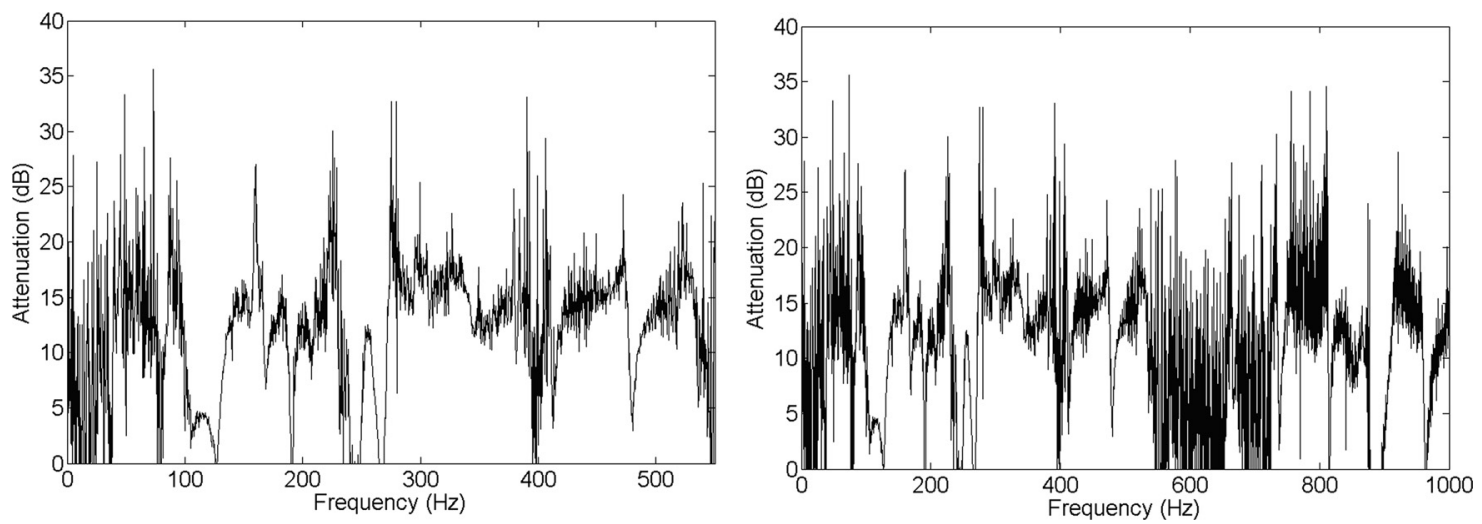

FIG. 2. Attenuation of the unwanted sound in the shielded domain. Left-hand graph is a zoom-in of the low frequency results.

shown in Fig. 2. In this initial experiment, wanted sound is not included, and a broadband linear unidirectional swept sine signal is used to generate a noise field. The signal is swept forward in frequency up to $1 \mathrm{kHz}$ with a rate of $380 \mathrm{~Hz} / \mathrm{s}$. The signal is generated through the signal generator with a sampling rate of $2560 \mathrm{~Hz}$ and resolution of 16 bits/sample. When the AS control system is applied to a duct where only an unwanted sound field propagates, it is found that the solution is able to achieve an attenuation of about $12-18 \mathrm{~dB}$ in the shielded domain over a large frequency range between 50 and $1 \mathrm{kHz}$ except at some points, mostly at anti-resonances. This is due to the fact that the sound level at anti-resonances is very low. Hence the error in the measurement is relatively high. The low attenuation between 580 and $700 \mathrm{~Hz}$ is another example of very small sound pressures at the boundary of the shielded domain at those frequencies when the boundary is close to the nodal plane of the predominant resonant mode. Overall, the result shows that the general solution which was used for the experiments with pure tone sources ${ }^{20}$ is also effective with broadband noise, as long as the sound field is strong enough to be measured accurately at the boundary of the shielded domain.

The sensitivity analysis (SA) has two objectives, i.e. to estimate the sensitivity to changes in the input parameters, and to identify the dominant sources of error (uncertainty) affecting the resulting attenuation. The investigation evaluates the quality of the control system, such as functionality and reliability in operation against uncertainties. The analysis is essential for the development of guidelines for the practical use of the method. The error sources in the AS method can be classified largely in two groups, one related to position and the other to time. For instance, errors in the measuring position, $\Delta x$, and the separation between loudspeakers forming a dipole, $\Delta d$, may exist in the realization of the controls. It is not uncommon that real environment does not allow for physical devices to be mounted at exactly the desired positions. These kinds of errors are concerned with the spatial aspect of the system. On the other hand, errors in time delay, $\Delta t$, and phase error, $\Delta \varphi$, can occur in digital signal processing or measuring equipment. In addition, changes of the input and output system responses with time can cause errors too, if the system is not controlled adaptively in realtime. The initial system parameters may also be changed by the introduction of the control sources. The purpose of the
SA is to prove the robustness of the control system due to systematic small errors. However, this analysis does not look into time varying errors.

Total deviation of the overall attenuation $\delta \eta$ can be estimated as follows: $\delta \eta=\sum\left|T_{i}\right| \cdot \delta n_{i}$, where the problem is assumed to be linear. Figure 3 shows the sensitivity $\left|T_{i}\right|$ with respect to the estimated changes in different variables $n_{i}$. The variables $n_{i}$ represent factors, namely $\Delta x, \Delta t, \Delta d$, and $\Delta \varphi$, that are perceived to have a strong influence on the accuracy of the experiment. $\Delta x$ is related to the size of the measuring microphone used in the experiment, in this case a quarter-inch microphone. $\Delta x$ is assumed to be half the diameter of the microphone head, i.e. $0.3175 \mathrm{~cm} . \Delta t$ is the minimum controllable time step which generally depends on the limited time resolution of the digital signal generator and the sampling frequency used in the measurement. For this calculation, the sampling frequency is assumed to be $2048 \mathrm{~Hz}$, which gives $\Delta t$ as $0.488 \mathrm{~ms}$. $\Delta d$ is the assumed error due to uncertainty in the actual distance between the centers of the loudspeakers composing the dipole source and is taken as $1 \mathrm{~cm}$ in this analysis. $\Delta d$ includes the uncertainty of the

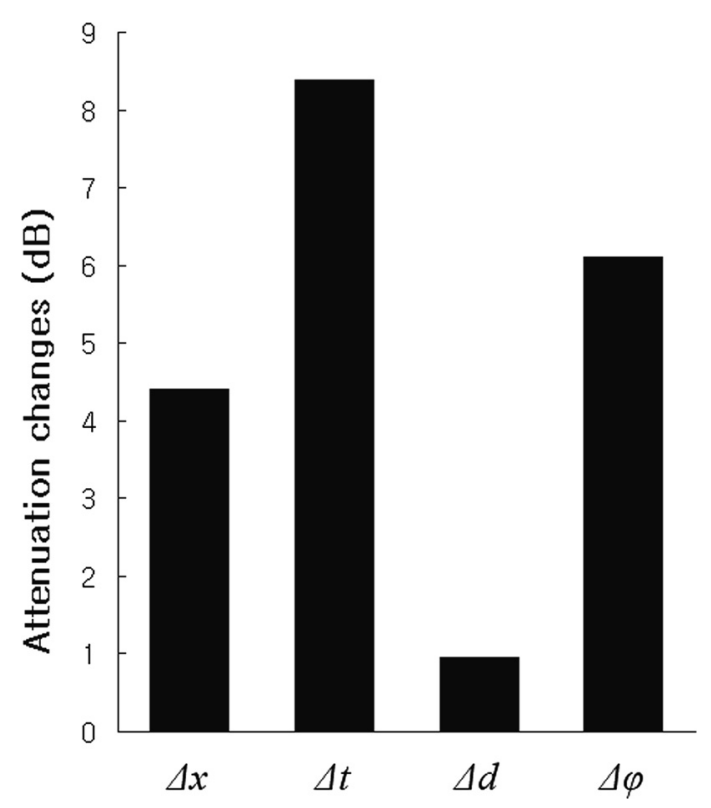

FIG. 3. Deviations of attenuation according to estimated changes in $\Delta x, \Delta t$, $\Delta d$, and $\Delta \varphi$. 
effective acoustic centers of the drivers composing the dipole source. The uncertainty in the overall phase $\Delta \varphi$, which is assumed to be a frequency independent error, up to $1 \mathrm{kHz}$ is assumed to be $1^{\circ}$ for the purpose of the analysis.

The result of the SA shows that the dominant parameters affecting the change of attenuation, when realistic values of uncertainty are used, are $\Delta t$ and $\Delta \varphi$, as can be seen in Fig. 3 . $\Delta \varphi$ is usually manifested also as a time delay in the time domain. Therefore, the time signatures describing the control sources have to be dealt with in high accuracy. A typical modern digital signal processing system is able to sample input data at frequency higher than $2048 \mathrm{~Hz}$, which corresponds to a $\Delta t$ of $0.488 \mathrm{~ms}$. The size of the measuring device used for measurement causes acceptable error in attenuation, as long as the sound field is measured as close as possible to the effective center of the control source unit. As stated above, the time delay error is the most dominant variable as compared to the other error parameters. These results are consistent with the theoretical SA performed in Ref. 28.

In further experiments described in later sections, broadband wanted sound fields are included to test the efficiency of the AS method in different configurations requiring preservation of wanted sound.

\section{Active control of multiple sound fields in multi-domain}

Figure 4 describes the experiment which demonstrates the possibility of using AS to preserve multiple wanted sounds in multi-domains. In the experiment, two shielded domains are defined in both left and right ends of the duct. Two different sources of the wanted sound are generated, one inside each shielded domain. In addition, a noise source is activated in the space between the two shielded domains. To test the capability of the method in more practical cases, human voice and a music track are used in the left- and right-hand shielded domain, respectively, to generate the broadband wanted sounds in the experiment. The wanted sound signals are captured from recorded audio tracks. To fit into the test frequency range of the duct, frequency contents

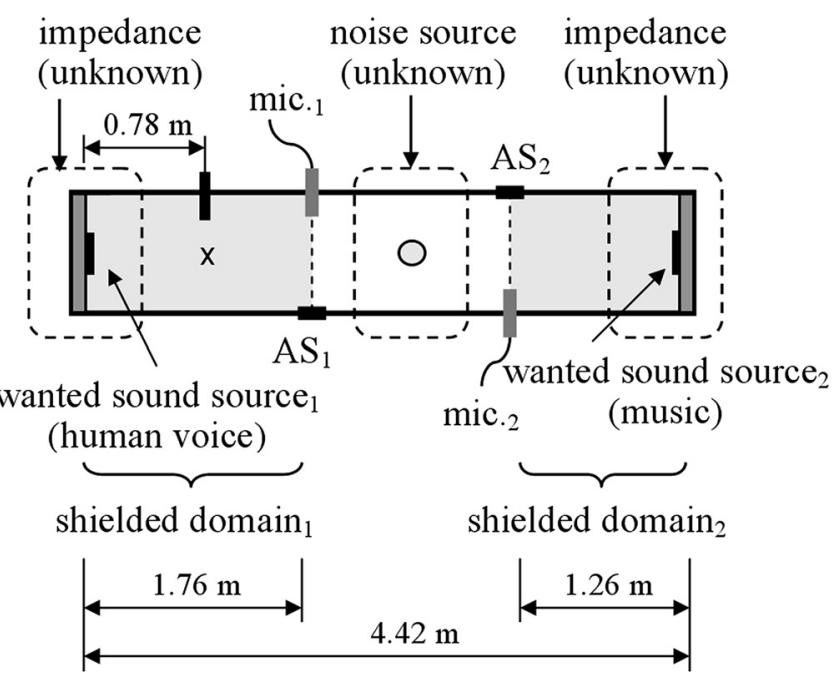

FIG. 4. Experimental setup for the test with two wanted sounds and one noise source in a multi-domain setting. of the wanted sound sources that are higher than $1 \mathrm{kHz}$ are attenuated by a low-pass filter. The wanted sounds are chosen to have practically different acoustic characteristics than the unwanted sound, which is generated by a white noise signal, in the experiment. The different time signatures of the output sound fields are illustrated further in this section.

To make the experimental model more general, the geometry of the system is designed to be asymmetric with respect to the size of the domains and location of the noise source. AS controls are mounted on the boundaries between the unwanted noise field and the shielded domains. To generalize the experiment further, and to take advantage of the potential-based method's ability to work without precise knowledge of system conditions, terminations with unknown finite impedance at both ends are used. In the experiment, the unknown impedance condition is implemented by putting approximately four inches thick generic fibrous sound absorbing material on the rigid plate at each end of the tube. The properties of the fibrous material are not known and are not needed in the potential-based approach.

To apply Eqs. (1) and (2), the study considers the shielded domains 1 and 2 together as one multiply connected domain $D$. Thus, the boundary of the domain $D$ contains two parts. The experiment convincingly demonstrates that the potential-based AS automatically extracts all the necessary information about the system and the unwanted noise itself from the measurements performed at each boundary. The source strengths of the control monopoles and dipoles are obtained by substituting the measured quantities, particle velocity $u_{o}$, and pressure $p_{o}$, of the total sound field at each boundary into the equations for the strengths of the monopoles and dipoles.

Figure 5 illustrates the change of the total sound pressure level in one of the shielded domains, at location $(x)$, specified in Fig. 4, before and after the control sources are activated on both boundaries, and shows the similarity between the result with AS activated and the original wanted sound in the frequency domain. The symbols - in Fig. 5 show the initial sound pressure distribution when the noise and wanted sounds are both on, while the control sources are still off. The symbols + represent the distribution of the net sound pressure when the noise is canceled out by the controls. The net sound pressure + can be compared with the wanted sounds, shown by the symbols $\bigcirc$ in Fig. 5. When the control sources are activated, the control system attains an overall attenuation of around $21 \mathrm{~dB}$ in both the left- and right-hand shielded domains. Moreover, the net sound pressure after the potential-based controls switched on generally resembles closely the original wanted sound pressure at each of the measurement positions in the shielded domains. The next figure shows a clearer picture with the same results presented in 1/3-octave bands.

Figure 6(a) shows the similarity between the original wanted sound pressure $\bigcirc$, and the result, $\Delta$ when the controls are switched on. In the experiment, a challenging condition is set up by introducing a significantly bigger unwanted sound pressure than the wanted one (about $10 \mathrm{~dB}$ higher), so that the results can give a reliable guidance of the attenuation that can be achieved in practice when the wanted sound has 


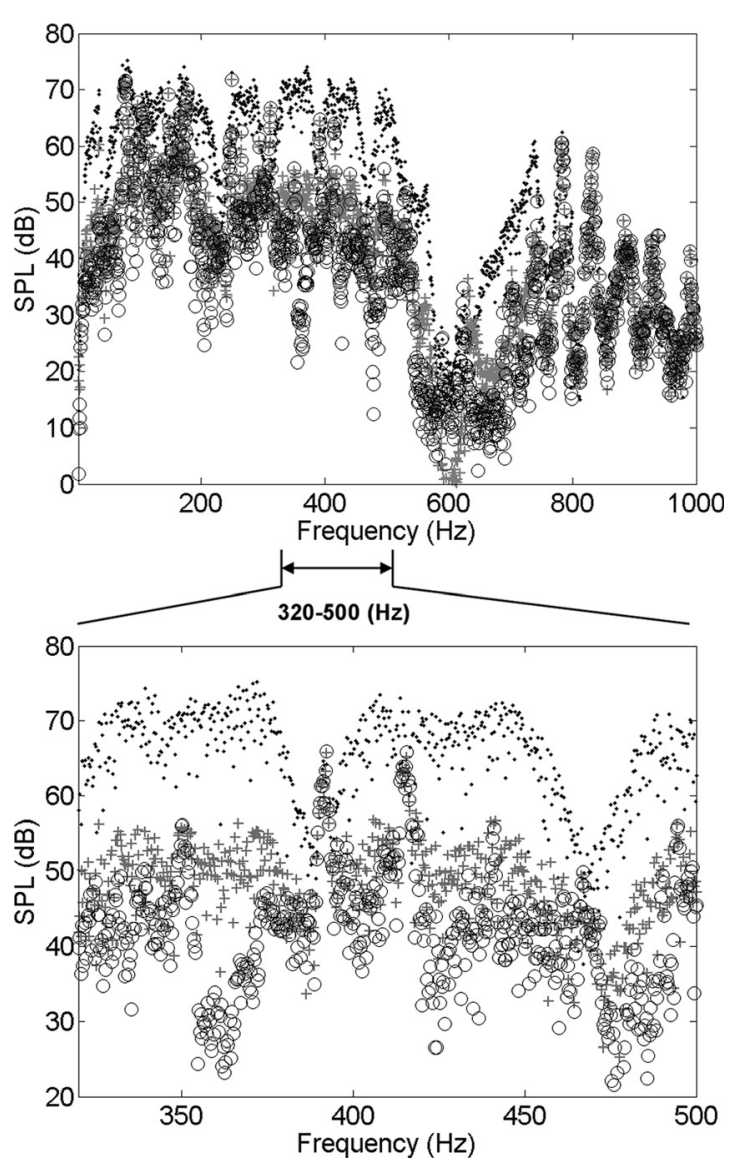

FIG. 5. Preservation of the wanted sound in one of the shielded domains; $\bigcirc$ : wanted sound pressure, $\bullet:$ the sound pressure of noise and wanted sound without control, and + : total sound pressure of noise and wanted sound, and controls. Bottom graph is a zoom-in of the result in the $320-500 \mathrm{~Hz}$ frequency range.

been seriously contaminated by strong unwanted noise. In spite of significant level differences between the unwanted noise and the wanted sound pressure, which are denoted by $*$ and $\bigcirc$, respectively, in each frequency band in Fig. 6(a), the wanted sound pressure is mostly preserved after the controls are switched on. Figure 6(b) shows the error between the result and the original wanted sound in decibel scale against the level difference between the unwanted noise and wanted sound pressure. Obviously, when the unwanted noise becomes stronger relative to the wanted sound, the error increases slightly due to the decrease in signal to noise ratio. However, even when the noise is up to $15 \mathrm{~dB}$ stronger, the errors is still less than about $1 \mathrm{~dB}$. When the difference is higher than $15 \mathrm{~dB}$, i.e. when the signal to noise ratio is below $-15 \mathrm{~dB}$, the error increases rapidly due to inherent errors in the measurement system.

To further support and enhance the experimental evidence of the preservation of the wanted sounds, the results are also studied in the time domain. Figure 7 illustrates the time signatures of data described above in Figs. 5 and 6.

The solid line in Fig. 7(a) shows the initial sound pressure when the noise and wanted sounds are both on, while the control sources are still off. Figure 7(b) represents the net sound pressure field when the noise is canceled out after the activation of the AS control sources. For comparison, the original wanted sound is separately measured at the same reference position when both the AS control and the unwanted noise sources have been turned off. This is shown in Fig. 7(c). The shielded result and the original wanted sound are overlaid in Fig. 7(d) for the time period between 2.2 and $2.3 \mathrm{~s}$ to give a clearer view. The figure shows clearly that, on the whole, the total sound field with the potentialbased control sources resembles closely the original wanted sound fields at each measuring position in the shielded domain. The similarity of the net sound field shielded by the AS control sources and the original wanted sound fields is also evaluated by the cross-correlation of the two time signatures $^{29}$ shown in Fig. 7. The cross-correlation of the wanted sound and the total sound pressure that consists of the unwanted noise and the wanted sounds without controls is at a maximum of 0.67 at zero time-lag. When the AS control sources are switched on, the cross-correlation of the wanted sound and the shielded total sound pressure (the unwanted noise, the wanted sounds, and the controls together) jumps to

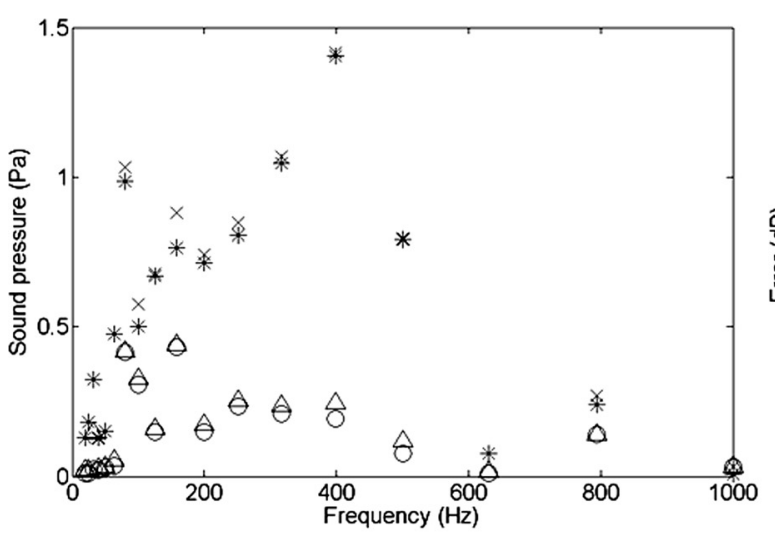

a)

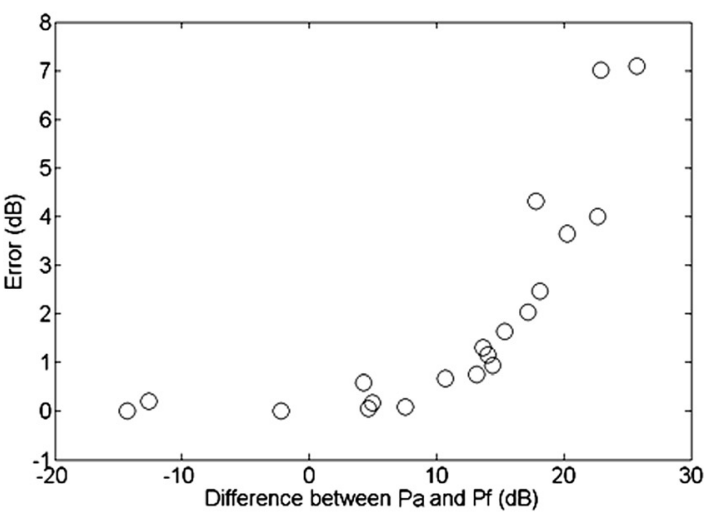

b)

FIG. 6. In a scale of one-third octave bands: (a) sound pressure distribution (overall noise about $10 \mathrm{~dB}$ higher than the wanted sound); $\bigcirc$ : wanted sound pressure, $\Delta$ : shielded total sound pressure (the sum of unwanted, wanted sound, and control outputs), $*$ : unwanted sound pressure, and $\times$ : the sum of unwanted and wanted sound pressure, and (b) errors between actively shielded results and the wanted sound against the difference between unwanted and wanted sound pressure levels. 


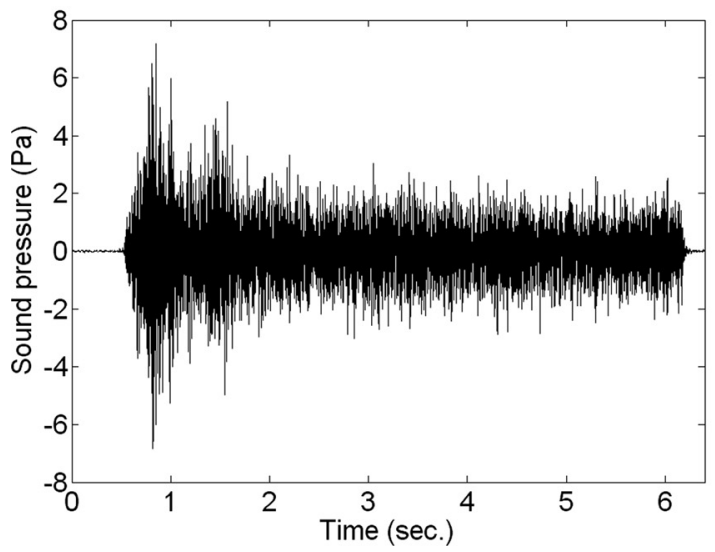

a)

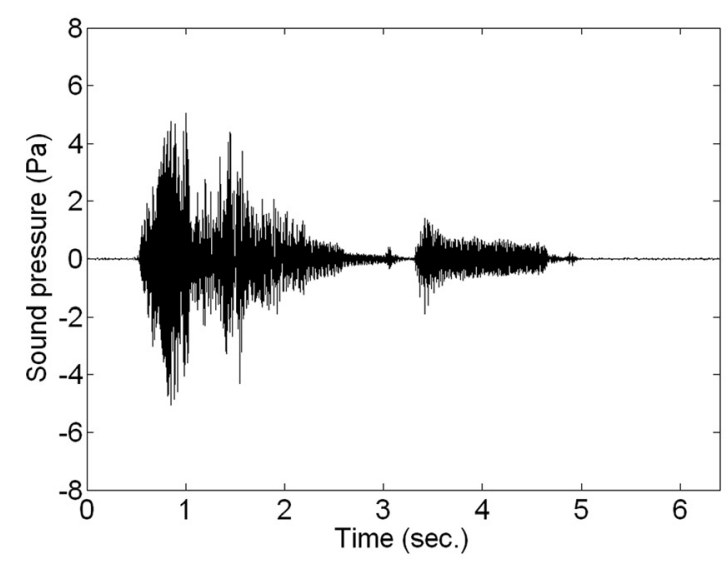

c)

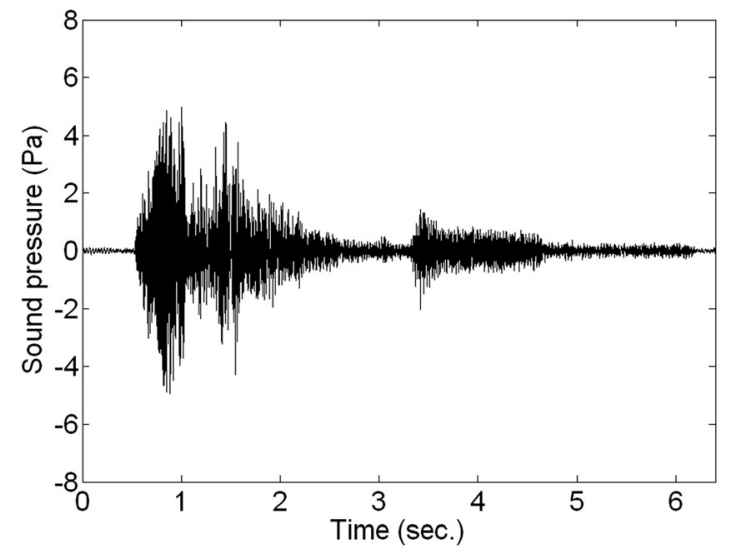

b)

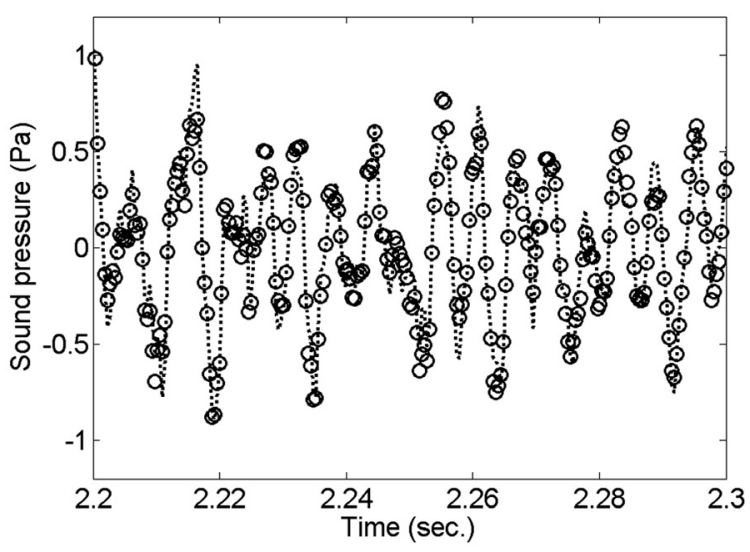

d)

FIG. 7. Sound pressure in time domain: (a) the sound pressure of noise and wanted sound without control, (b) shielded total sound pressure (noise, wanted sounds, and controls), (c) wanted sound pressure, and (d) ... wanted sound pressure, and $\bigcirc$ : shielded total sound pressure.

0.99 at the same point of time-lag. The ideal cross-correlation of two identical signals is 1.0. This is almost achieved in the experiment, which shows that the shielded net sound field with the controls on matches the original wanted sound field very well. The experiment clearly proves that wanted sounds can be very effectively protected by the active controls based on the proposed method even in multi-domains where wanted sounds from different shielded domains can also interfere with each other, while unwanted noise is significantly suppressed by the AS control sources.

Despite the difficulty in dealing with multiple control sources and the complexity of multi-domains, the results in this section show better efficiency in the overall attenuation and preservation of the wanted sounds, when compared with the result discussed in the previous section. One of the main reasons can be found in the design of the experimental model. That is, the terminations are more absorptive in this multi-domain experiment, which damped the tube resonances. At resonances and anti-resonances the result is much more sensitive to measurement errors due to large changes in the sound pressure, ${ }^{28}$ see also the SA in Sec. III B. By damping out the resonances, this sensitivity to error is significantly reduced. The other reason for the better result is that the accuracy of the control system has also been improved by explicitly taking some of the causes of inherent measure- ment errors into account. For instance, the phase distortion caused by a time delay in the digital-to-analog converter used is corrected for in this latest measurement. According to the SA in Sec. III B, it has been shown that the control system is most sensitive to time delay errors. Correcting for this error thus improves the result significantly. All these together make a significant contribution to the stability of the whole system and the repeatability of the measurements.

\section{CONCLUSIONS}

The practicality of AS based on the method of difference potentials has been experimentally validated with broadband acoustic sources in a variety of one-dimensional bounded domains. Unlike previous experiments with pure tone sources, the sound fields generated by broadband signals in the experiments presented in this paper include substantial resonances and anti-resonances in the system. Through these experiments, it has been shown that attenuation from 15 to $20 \mathrm{~dB}$ can be achieved even at resonances in the shielded domains, whether or not wanted sounds exist in the same space. Similar to other existing AS methods, the effectiveness of the control solution based on the difference potentials method are most sensitive to time delay errors especially at resonances. Apart from the practical difficulties 
associated with this high sensitivity at resonances, the present experiments show that the method can provide an effective solution not only at non-resonance regimes but also through a full continuous broadband spectrum of frequency that includes resonances. However, in practice, it is difficult to find an accurate solution at strong anti-resonances when the physical values of the total sound field to be measured at the boundary are too small to be measured with sufficient accuracy. This is limited by the dynamic range of the measurement probe and the very low signal to noise ratio at strong anti-resonances.

The level of attenuation found in the result of the experiments is similar to the those achieved by other existing conventional AS methods when broadband sources are used in a one-dimensional enclosure. In addition to the significant suppression of noise, the proposed method has been shown to also effectively preserve the wanted sounds separately from the total fields of noise and wanted sounds, even when there are multiple shielded domains with interfering wanted sounds from different domains, and that the system characteristics are not known. The results clearly demonstrate the potential advantages of the method in practical applications under these conditions.

This paper has shown that the proposed approach can be realized provided that the contribution of the control sources to the input data can be separated. An obvious question to follow up is how to obtain such separation in practice. Theoretically, it has been proven in Ref. 18 that this can be done via a modification of the solution presented by Eq. (6). It requires additional on-line calculations of surface potentials, which can be efficiently carried out via the method of difference potentials. However, the implementation of the algorithm is far from trivial and will be an objective of our future research.

\section{ACKNOWLEDGMENTS}

The research was supported by the Engineering and Physical Sciences Research Council (EPSRC) under the project codes, GR/T26825/01 and GR/T26832/01.

${ }^{1}$ P. A. Nelson and S. J. Elliott, Active Control of Sound (Academic Press, San Diego, CA, 1992), pp. 116-122, 143-146, 311-378.

${ }^{2}$ R. K. Kincaid, S. L. Padula, and D. L. Palumbo, "Optimal sensor/actuator locations for active structural acoustic control," AIAA Paper 98-1865, in Proceedings of the 39th AIAA/ASME/ASCE/AHS/ASC Structures, Dynamics and Materials Conference, Long Beach, CA (1998).

${ }^{3}$ R. K. Kincaid and K. Laba, "Reactive tabu search and sensor selection in active structural control problems," J. Heuristics 4, 199-220 (1998).

${ }^{4}$ M. Hodgson, J. Guo, and P. Germain, "Active local control of propelleraircraft run-up noise,” J. Acoust. Soc. Am. 114(6), 3201-3210 (2003).
${ }^{5}$ V. S. Ryaben'kii, “A difference shielding problem,” J. Funct. Anal. Appl. 29(1), 70-71, 1995.

${ }^{6}$ V. S. Ryaben'kii, Method of Difference Potentials and its Applications (Springer-Verlag, Berlin, 2002), pp. 515-522.

${ }^{7}$ M. J. M. Jessel and G. A. Mangiante, "Active sound absorbers in an air duct," J. Sound Vib. 23(3), 383-390, 1972.

${ }^{8}$ G. A. Mangiante, “Active sound absorption,” J. Acoust. Soc. Am. 61(6), 1519-1522, 1977.

${ }^{9}$ G. Canevet, "Active sound absorption in air conditioning duct," J. Sound Vib. 58(3), 333-345, 1978.

${ }^{10} \mathrm{G}$. Mangiante, "The JMC Method for 3D active sound absorption: a numerical simulation," Noise Control Eng. J. 41(2), 339-345, 1993.

${ }^{11}$ V. S. Ryaben'kii, S. V. Tsynkov, and S. V. Utyuzhnikov, "Inverse source problem and active shielding for composite domains," J. Appl. Math. Lett. 20(5), 511-515, 2007.

${ }^{12}$ A. W. Peterson and S. V. Tsynkov, "Active control of sound for composite regions,” SIAM J. 67(6), 1582-1609, 2007.

${ }^{13}$ J. Loncaric, V. S. Ryaben'kii, and S. V. Tsynkov, "Active shielding and control of noise," SIAM J. 62(2), 563-596, 2001.

${ }^{14} \mathrm{~S}$. V. Tsynkov, "On the definition of surface potentials for finite-difference operators," J. Sci. Comput. 18, 155-189, 2003.

${ }^{15}$ V. S. Ryaben'kii, S. V. Utyuzhnikov, and A. Turan, "On the application of difference potential theory to active noise control," J. Adv. Appl. Math. 40(2), 194-211, 2008.

${ }^{16}$ V. S. Ryaben'kii, and S. V. Utyuzhnikov, "Active shielding model for hyperbolic equations,” IMA J. Appl. Math. 71(6), 924-939, 2006.

${ }^{17}$ Yu. V. Egorov and M. A. Shubin, Foundations of the Classical Theory of Partial Differential Equations (Springer, Berlin-London, 1992).

${ }^{18}$ S. V. Utyuzhnikov, "Generalized Calderon-Ryaben'kii's potentials," IMA J. Appl. Math. 74(1), 128-148, 2009.

${ }^{19} \mathrm{~S}$. V. Utyuzhnikov, "Active wave control and generalized surface potentials,” J. Adv. Appl. Math. 43(2), 101-112, 2009.

${ }^{20}$ H. Lim, S. V. Utyuzhnikov, Y. W. Lam, A. Turan, M. R. Avis, V. S. Ryaben'kii, and S. V. Tsynkov, "Experimental validation of the active noise control methodology based on difference potentials," AIAA J. 47(4), 874884, 2009.

${ }^{21}$ J. Loncaric and S. V. Tsynkov, "Optimization of acoustic source strength in the problems of active noise control," SIAM J. Appl. Math. 63, 1141$1183,2003$.

${ }^{22}$ V. S. Ryaben'kii, S. V. Tsynkov, and S. V. Utyuzhnikov, "Active control of sound with variable degree of cancellation," J. Appl. Math. Lett. 22(12), 1846-1851, 2009.

${ }^{23}$ A. J. Zuckerwar and R. W. Meredith, "Radiation losses in resonant tubes," J. Acoust. Soc. Am. 70, 879-885, 1981.

${ }^{24}$ C. H. Hansen and S. D. Snyder, Active Control of Noise and Vibration (E. \& F. N. Spon, London, 1997), pp. 555-556.

${ }^{25}$ S. Uosukainen and V. Valimaki, JMC Actuators and Their Applications in Active Attenuation of Noise in Ducts (VTT Publications, 341, VTT Building Technology, ESPOO, 1998), p. 100.

${ }^{26}$ S. Uosukainen, "Modified JMC method in active control of sound," Acust. Acta Acust. 83, 105-112, 1997.

${ }^{27}$ K. Anai, T. Shiki, Y. Hiraguri, and K. Fujimoto, "Improving sound insulation capability at a ventilation opening using active noise control: Improving sound insulation performance degraded by living sound," in Proceedings of Inter-Noise 2008, Shanghai, Paper No.0252 (2008).

${ }^{28}$ S. V. Utyuzhnikov, "Nonstationary problem of active sound control in bounded domains," J. Comput. Appl. Math. 234(6), 1725-1731, 2010.

${ }^{29}$ R. B. Randall and B. Tech, Frequency Analysis (Brüel\& Kjær, Denmark, 1987), pp. 252-253. 